Glucocorticoids protect the heart

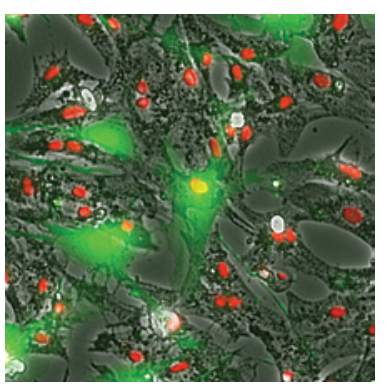

The ability of glucocorticoids to mediate cardioprotective effects in the setting of acute myocardial ischemia/reperfusion has largely been attributed to their antiinflammatory effects. However, Tokudome and colleagues have now determined that glucocorticoid activation of the $\mathrm{PGD}_{2}$ biosynthesis pathway also has cardioprotective effects against ischemia/reperfusion injury in rodent hearts (1477-1488). In vitro analysis of rat cardiomyocytes indicated that glucocorticoids, acting via the glucocorticoid receptor (GR), increased expression of the genes encoding COX2, cytosolic phospholipase A2, and lipocalin-type prostaglandin D synthase (L-PGDS), all of which participate in $\mathrm{PGD}_{2}$ biosynthesis. Consistent with this, glucocorticoids stimulated production of $\mathrm{PGD}_{2}$ in cultured rat cardiomyocytes and in the adult mouse heart when administered i.p. Importantly, glucocorticoids reduced ischemia/reperfusion injury and infarct size in isolated Langendorff-perfused mouse hearts and an in vivo mouse model of ischemia/reperfusion injury, respectively. In both cases, protection was dependent on L-PGDS. The authors therefore suggest that GR-selective agonists might be beneficial in humans following myocardial infarction, acting to limit infarct size and improve mortality.

\section{Keeping sepsis under control}

An underlying cause of death from sepsis is multiple organ failure due to deposition of microthrombi in the vasculature. Prior to this, inflammation causes vascular leakage, which leads to systemic activation of the hemostatic system and thus disseminated intravascular coagulation (DIC) and microthrombi in the vasculature. Washington and colleagues have now generated data in humans and mice indicating that triggering receptor expressed on myeloid cells-like (TREM-like) transcript-1 (TLT-1) is a critical regulator of inflammatory activation of hemostasis during sepsis (1489-1501). In humans, the authors found that, unlike healthy individuals, patients with sepsis had substantial amounts of soluble TLT-1 (sTLT-1) in their plasma and that its presence correlated with DIC. Consistent with sTLT-1 having an active role in DIC, human sTLT-1 bound to fibrinogen and augmented platelet aggregation in vitro. Analysis of mice lacking TLT-1 confirmed a role for sTLT-1 as a regulator of hemostasis, platelets from the mice were impaired in their abil-

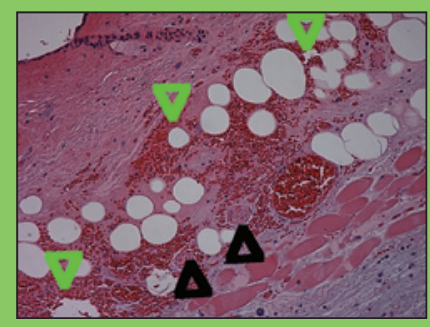
ity to aggregate in vitro and in vivo (as assessed by increased bleeding times), and the mice were more susceptible to both challenge with LPS and inflammatory hemorrhage. The authors therefore suggest that TLT-1 helps maintain vascular integrity and contain the inflammatory response in the initial phases of sepsis.

\section{ceacamo restricts} adenoviral oncolytic potential

One new approach being developed for the treatment of cancer that is resistant to standard therapies is the use of oncolytic adenoviruses. Although promising clinical results have been obtained in some settings, in many cases the therapies have had little impact. Wang and colleagues have now identified one reason for this lack of efficacy: carcinoembryonic antigen-related cell adhesion molecule 6 (CEACAM6) decreases the ability of a cancer cell to be infected by adenovirus (1604-1615). Initial analysis of human pancreatic cancer cell lines revealed that expression of CEACAM6 correlated inversely with sensitivity to adenovirus and that ectopic overexpression of CEACAM6 in adenovirus-sensitive cell lines substantially decreased their susceptibility to adenovirus infection. Conversely, siRNA-mediated knockdown of CEACAM6 expression increased cancer cell susceptibility to adenovirus infection both in vitro and in a mouse xenograft model, in which marked antitumor effects of an oncolytic adenovirus were observed. Mechanistically, CEACAM6 was found to block adenoviral cytoplasmic trafficking to the nucleus of human pancreatic cancer cells by interfering with the Src signaling pathway and thereby downregulating expression of cytoskeletal proteins. The authors therefore suggest that determining whether a tumor expresses CEACAM6 might provide a way to predict the response of a patient to adenovirus-based therapies. 